\title{
Permeability and Porosity of Two-Dimensional Porous Media Microchannels using Lattice Gas Cellular Automata
}

\author{
Siti Nurul Khotimah ${ }^{1 *}$, Bergas Bimo Branarto ${ }^{2}$, Sparisoma Viridi $^{1}$ and Idam Arif ${ }^{1}$ \\ ${ }^{I}$ Nuclear Physics and Biophysics Research Division \\ ${ }^{2}$ Physics Study Program \\ Faculty of Mathematics and Natural Sciences, Institut Teknologi Bandung \\ Jalan Ganesa 10, Bandung 40132, Indonesia
}

Received: 2 February 2012, Revised: 15 June 2012, Accepted: 29 June 2012

\begin{abstract}
In this study, lattice-gas cellular automata were used to solve the flow of incompressible Newtonian-fluid in porous media microchannels. We discuss fluid flow between two stationary parallel plates. By applying a constant pressure gradient, volumetric flux was determined as a function of time until a steady condition is achieved. For steady laminar flow, its velocity profile is parabolic. For flow in porous media between two stationary parallel plates, the results show that medium permeability depends on porosity and obstacle configurations. For a single obstacle, the permeability is a parabolic function with respect to positions of an obstacle in the direction perpendicular to the flow. The permeability is smallest when the obstacle is at the central line along the flow. A maximum permeability may be achieved when the obstacles attached to the channel wall. Other obstacle structures give lower permeability, even zero permeability for dead end microchannels.
\end{abstract}

Keywords: lattice-gas automata, fluid dynamics, flow stream, porous media

\section{INTRODUCTION}

In the concepts of cellular automata, each automaton in its internal state will behave as a processor that receives inputs, processes the inputs, and determines the next internal state and its outputs [1]. Therefore the computation method based on artificial neural network [2] can also be included as cellular automata method. This method enables to give solution in many problems that are difficult to be worked out conventionally.

In general, a fluid molecule has a linear size in the order of nanometer. A molecule occupies one lattice site in triangular lattice in the Lattice Gas cellular automata. This study discusses fluid flows in channels having linear dimensions of $160 \times 72$ lattice sites. We call micrometer-sized channels as microchannels.

Parallel plate flow is an object of extensive study because it is a simple model for flow through a

\footnotetext{
Corresponding author.

E-mail address: nurul@fi.itb.ac.id
}

crack or joint of a rock $[3,4]$. This flow is a 2-D channel flow since flow in the third dimension is invariant. Micrometer-sized media between two parallel plates are then selected as two-dimensional media microchannels. Obstacles in this media are used to simulate two-dimensional porous media microchannels.

Using lattice-gas cellular automata (LGA), we study fluid flow in micrometer-sized media between two stationary parallel plates caused by an applied pressure gradient. Volumetric flux is computed as a function of time until the flow achieves a steady state condition [3]. This paper reports the velocity profile of the flow at a steady state condition and also investigates the dependency of medium permeability on porosity and arrangement of obstacles.

\section{THEORITICAL BACKGROUND}

For steady incompressible laminar flow in porous media, Henri Darcy discovered experimentally that the volumetric flux $q$ is 
proportionally linear with the applied pressure gradient $d P / d x$ as follows [3].

$$
q=\frac{k}{\mu}\left(-\frac{d P}{d x}\right)
$$

where $k$ is permeability the medium and $\mu$ is dynamic viscosity of the fluid.
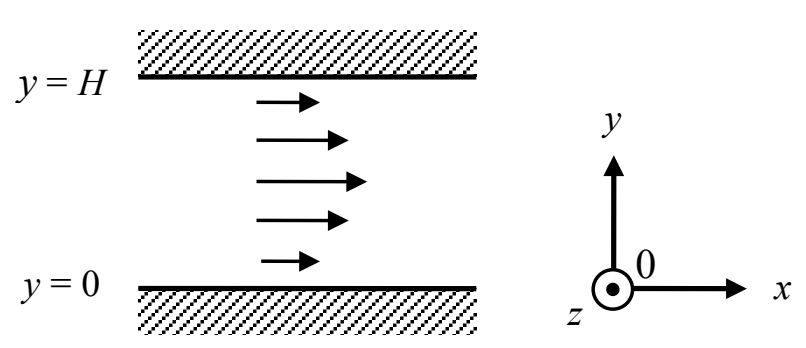

Figure 1. Two parallel plates at $y=0$ and $y=H$.

Analytical solution for steady incompressible laminar fluid flow between two stationary parallel plates has been known [5]. At a constant pressure gradient, velocity profile for laminar flow in Figure 1 is a parabolic curve, i.e.

$$
u(y)=\frac{1}{2 \mu}\left(-\frac{d P}{d x}\right)\left[y H-y^{2}\right]
$$

and the volumetric flux is proportional to the square of channel width:

$$
q=-\frac{H^{2}}{12 \mu} \frac{\Delta P}{L}
$$

where $u$ is the velocity of the fluid in $x$-direction, $\Delta P=P(x=L)-P(x=0)$ is pressure difference, $H$ is channel width, and $L$ is the channel length.

The volumetric flux $q$ at steady state is related with the velocity of the fluid in $x$-direction $u$ by porosity $\phi$ of the medium as given in this equation [3]

$$
q=\phi u
$$

where porosity $\phi$ is defined as void regions divided by the total region.

\section{THE LATTICE-GAS CELLULAR AUTOMATA}

LGA is introduced by Frisch, Hasslacher, and Pomeau in 1986 [6]. The automata are distributed on the nodes of a periodic triangular lattice. The automata change their states simultaneously by parallel iteration. The lattice-gas model is constructed of identical particles that move from node to node on a triangular lattice, colliding when they meet, and always obey the fundamental physical principles, i.e. conserving particle number and momentum. Particles of unit mass move with unit speed in the direction given by

$$
c_{i}=\left[\cos \left(\frac{2 \pi i}{6}\right), \sin \left(\frac{2 \pi i}{6}\right)\right] \quad i=1,2, \ldots, 6
$$

Any particle that reaches a material point is reflected back with its negative velocity. This rule is used to fulfill the non-slip boundary conditions. The lattice in the model is infinite, but in practice always has edges. Therefore periodic boundary condition is applied so that the left end of the lattice for medium in Figure 1 is connected the right end.

The units for kinematics quantities are as follows. Length is in lattice units (l.u.), i.e. the distance between two nearest nodes. Time step (t.s.) is the discrete time unit required for the update of the entire automata. One mass unit (m.u.) is the mass of a single particle. Therefore, velocity is in lattice units per time step (1.u./t.s.) and volumetric flux has the same unit as velocity. Pressure is in (m.u.) $\times(\text { l.u. })^{-1} \times(\text { t.s. })^{-2}$. The permeability and the dynamic viscosity are in (l.u. $)^{2}$ and (m.u.) $\times(\text { l.u. })^{-1} \times(\text { t.s. })^{-1}$ respectively.

\section{NUMERICAL TECHNIQUES}

\section{Lattize size, initial and boundary conditions}

The lattice size for this 2-dimensional flow in Figure 1 is $L \times H$. There are $n_{x}$ automata in $x$ direction so that $L=n_{x}$ 1.u. and $n_{y}$ automata $y$ direction so that $H=n_{y} \frac{\sqrt{3}}{2}$ l.u. We use $n_{x}=160$ and $n_{y}=72$ automata. The previous studies in LGA show that mean free path in lattice gas is approximately about 5-8 automata [3,7]. In this study, the pores have sizes more then the mean free path.

The density is 2.4 particles per site. At initial condition $t=0$, a number of input particles $\left(2.4 \times n_{x}\right.$ $\times_{n_{y}}$ ) are distributed in the lattice randomly both their positions and velocity directions. Particles that leave the lattice at $x=L$ reenter the lattice at $x=0$ and particles that leave at $x=0$ reenter at $x=L$. This periodic boundary condition is often used to simulate a large system by modelling a small part 
that is far from its edge. This condition also maintains constant particle number in the lattice. Any particle that reaches a material point is reflected back with its negative velocity.

\section{Pressure gradient}

A pressure gradient was created by changing the $x$-component of momentum particles at $x=0$ each time step during the experiment and no forces are applied at $x=L[3]$ :

$$
\frac{d P}{d x}=\frac{\Delta P}{L}=\frac{P(x=L)-P(x=0)}{L}=-\frac{n_{y} f_{x}}{H L}
$$

where $n_{y} f_{x}$ is total change in the $x$-component of momentum from particles distributed in $n_{y}$ automata at $x=0$ is the average change in the $x$ component of momentum at a single point at $x=0$. In this study, we use $f_{x}=0.0069$ which is generated from total change of 0.5 in the $x$ component of momentum each time step to give a pressure gradient of $5.012 \times 10^{-5}$.

\section{Velocity profile}

For a constant pressure gradient, the volumetric flux was plotted as a function of time until its value does not change significantly with time. When system has achieved its steady condition, velocity profile is then determined. Average $x$-component of velocity per particle is presented as a function of $y$. In order to obtain better data quality, we need to average the results from 10 runs with different randomness of particles in the initial condition. The data are reported in the averages \pm their standard deviations. From the result of velocity profile, the viscosity of the fluid can be determined.

\section{The obstacle's position-dependent medium permeability}

Using a square obstacle in the medium, permeability of medium is calculated from equation (1) with input parameter is pressure gradient and output data is volumetric flux at steady state. The position of the obstacle is varied along the $y$ direction. The particles are grouped into $8 \times 8$ automata to have one representative particle which is drawn as an arrow to give a description in macroscopic scale.

\section{The obstacles's configuration-dependent medium permeability}

The dependency of medium permeability on the porosity of medium is studied. The porosity of the medium is varied on the configurations of the obstacles. Therefore, this section aims to study the permeability of the medium on the obstacles's configurations.

\section{RESULTS AND DISCUSSION Velocity Profile}

In fluid mechanics, the velocity of viscous fluid at solid boundaries is zero and the flow is fastest at the center. Velocity profile for steady parallel-plate flow is shown in Figure 2. The velocity profile is parabolic. Using Equation (2) for the result of this velocity profile, we found that the viscosity of the fluid was 0.143 . This result agrees for laminar flow. This parabolic velocity profile has also been reported by previous studies using greater values for pressure difference with $f_{x}=0.025$ [3] and $f_{x}=0.0125[8]$.

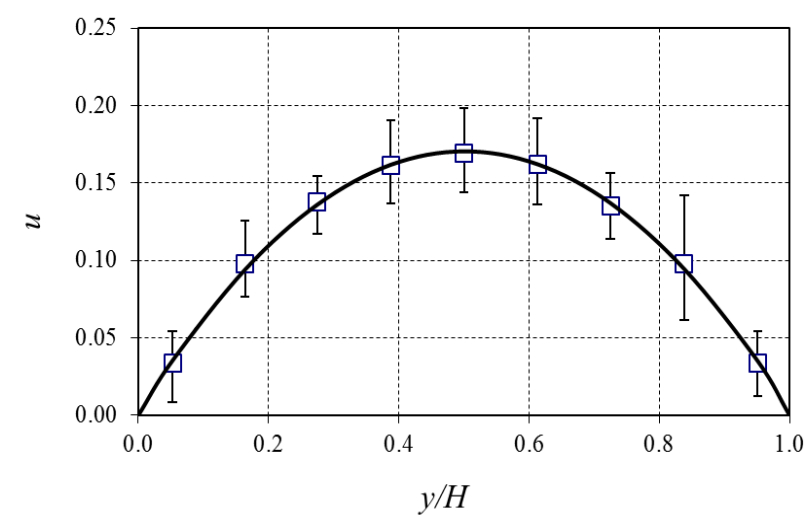

Figure 2. Numerical result for $u(y)$ compared to the theoretical parabolic profile $(L=16-, H=72 \sqrt{3} /$ 2 and $\left.f_{x}=0.0069 ; R^{2}=0.998\right)$

\section{The obstacle's position-dependent medium permeability}

Using this low pressure gradient, a laminar flow is obtained. Threfore, the Darcy equation (1) can be applied providing the pore size is larger than mean free path in lattice gas. Figure 3 shows variations in position of a single obstacle along the channel width. This was made to observe its effect on permeability of medium. At the same porosity, it is found that permeability of medium is highest when the obstacle is located at the edge of the channel. In contrast, permeability of medium is lowest when the obstacle is in the middle of the channel as shown in Figure 4.

Figure 2 shows that the flow is fastest at the center. Therefore, if an obstacle is placed in the center then this will impact most on the decrease in 
volumetric flux. Conversely, if the obstacle is placed on the edge then this gives the smallest impact on the decline of the flux because the velocity in this area is small. Figure 4 shows that medium permeability for flow in $x$-direction is a parabolic function with respect to the positions of an obstacle in $y$-direction.
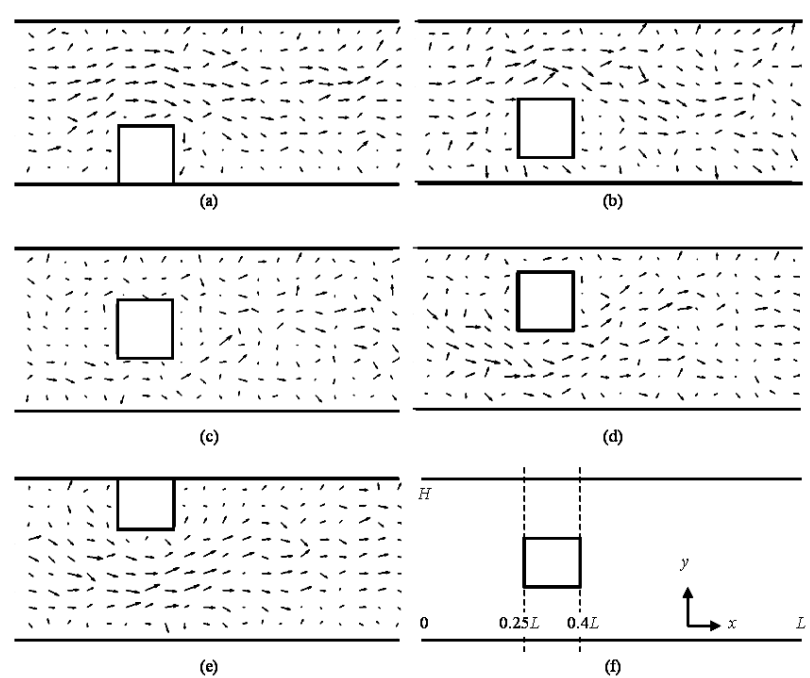

Figure 3. (a) - (e) Position of a square obstacle is varied in $y$-direction and ( $\mathrm{f}$ ) placed at $x$

between $0.25 \mathrm{~L}$ to $0.4 \mathrm{~L}$.

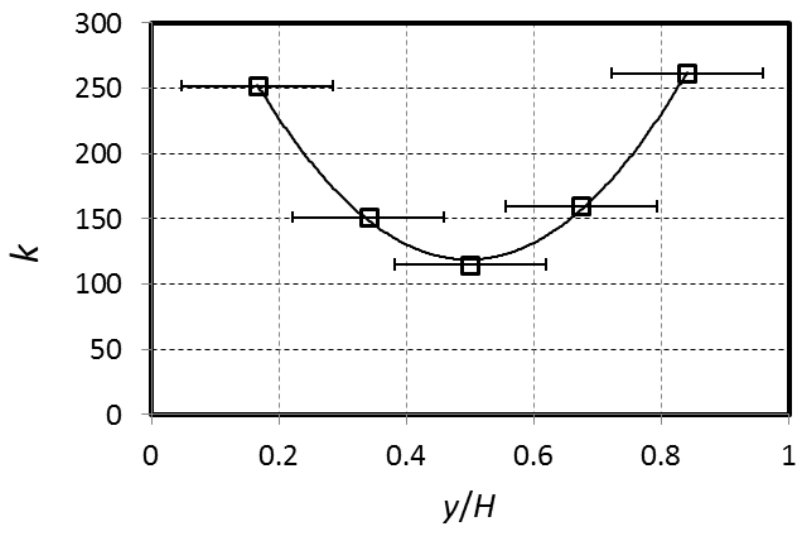

Figure 4. Permeability of medium for flow in x-direction is related to the positions of an obstacle in y-direction.

\section{The obstacles's configuration-dependent medium permeability}

Figure 8 summarizes ratio of the permeability $k$ of medium with respect to its maximum value $k_{0}$ versus porosity for 11 different shapes and arrangements of obstacles in a microchannel as illustrated in Figures 5-7. Figure 8 is similar to the data for conductivity coefficient of 23 rock samples if it is plotted with respect to porosity in the paper by Jan Šperl and Jiřina Trčková (2008) [9]. For twodimensional disordered porous medium, Reyes et al (1999) obtained a power-law dependence of permeability as a function of porosity [10]. It is identified from Figure 8 that there are 3 different clusters in grouping the relation between permeability and porosity.

First, permeability seems linearly related to porosity. This is appropriate for obstacle configurations shown in Figures $5 \mathrm{c}$ and $5 \mathrm{~d}$ which have symmetry with respect to the central line along the flow and the obstacles are attached to the channel walls. Second, permeability varies at the same porosities. At this second group, the different configurations of the obstacles affect the flow. Among configurations with $\phi=0.82$ (Figures 6c, 6d and Figure7), Figure $6 \mathrm{c}$ has the highest permeability since the obstacles are near to the channel walls where the flow is very slow. In contrast, permeability will be lower for porous medium with obstacles closer to the center of flow. Third, zero permeability for medium having total dead end as illustrated in Figure 6b. For this medium, flow rate is zero whatever the ratio of void to the total region.
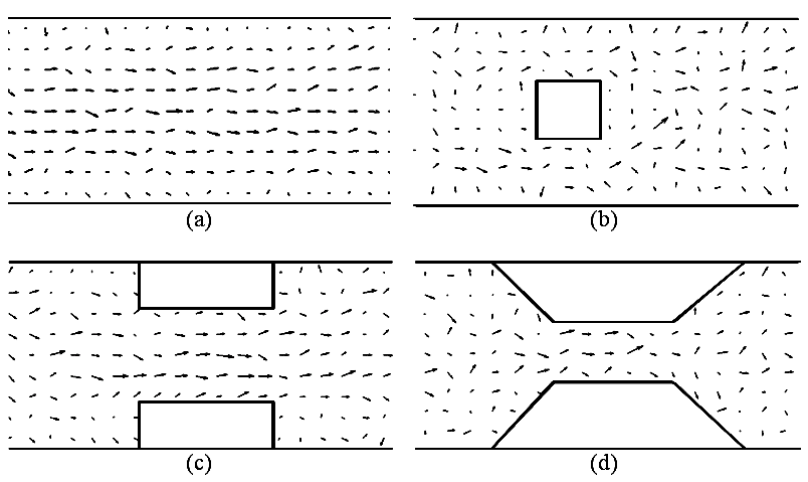

Figure 5. Some examples of obstacle configurations inside a microchannel:

(a) $\phi=1.0$, (b) $\phi=0.96$, (c) $\phi=0.91$, and (d) $\phi=0.79$.

In general, Figure 8 presents that there is maximum permeability which may be achieved when the obstacles attached to the channel wall. For other obstacle configurations, permeability will be lesser and even zero. The qualitative results from LGA may explain the dependency of permeability on porosity and the configurations of obstacles in microchannels. 

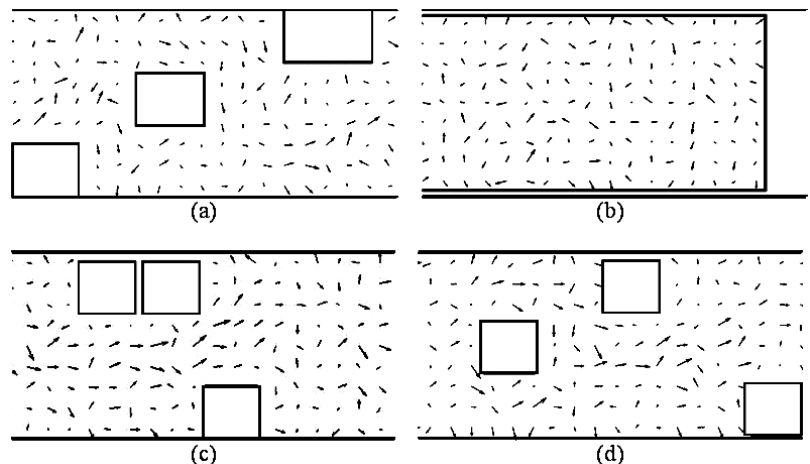

Figure 6. Some examples of obstacle configurations inside a microchannel: (a) $\phi=0.76$, (b) $\phi=0.85$, (c) $\phi=0.82$, and (d) $\phi=0.82$.
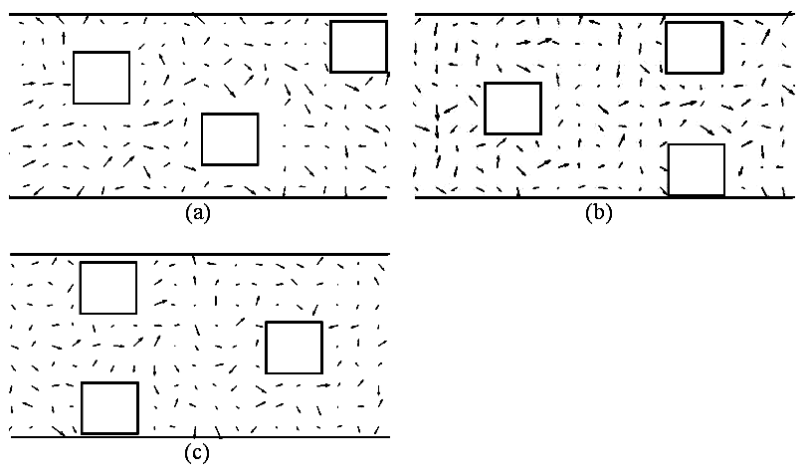

Figure 7. Some examples of obstacle configurations inside a microchannel at $\phi=0.82$

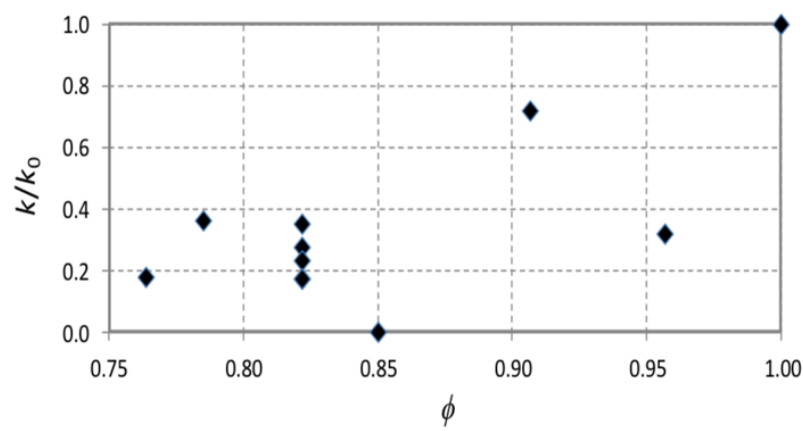

Figure 8 . Permeability of medium $k / k_{o}$ versus porosity $\phi$ for several obstacle configurations as illustrated in Figures 5-7.

\section{CONCLUSION}

By applying a constant pressure gradient, velocity profile is parabolic for steady laminar flow. This LGA results agree with the theory. Using this low constant pressure gradient, medium permeability depends on porosity and obstacle configurations. For a single obstacle, permeability is a parabolic function with respect to positions of the obstacle in direction perpendicular to the flow. The permeability is smallest when the obstacle is at the center of the flow.
The permeability of the medium achieved its maximum when obstacles attached to the channel walls. Other structures give lower permeability, especially for medium having obstacle located at the center of the flow, and even zero permeability for dead end channels.

\section{ACKNOWLEDGMENT}

Authors would like to thank to Nuclear Physics and Biophysics Research Division, Faculty of Mathematics and Natural Sciences Insitut Teknologi Bandung for the friendly discussion atmosphere.

\section{REFERENCES}

[1] G. Weisbuch, Complex System Dynamics, an Introduction to Automata Networks, Lecture Note Volume II, Santa FE Institute, Studies in the Science of Complexity, Addison-Wesley Publish. Co., 1991, pp. 7-9.

[2] R.C. Eberhart, and R.W. Dobbins, Neural Network PC Tools, Academic Press, 1990, pp.9-13.

[3] D. H. Rothman, Cellular-Automaton Fluids: A Model for Flow in Porous Media, Geophys. 53(4), 509-518 (1988).

[4] S.R. Brown, Fluid Flow through Rock Joints: the effect of surface roughness. J. Geophys. Res. 92 (B2), 1337-1347 (1987)

[5] W. J. Duncan, A. S. Thom, and A. D. Young, Mechanics of Fluids, $2^{\text {nd }}$ ed. ELBS and Edward Arnold Publ. Ltd., 1971, pp. 156-162.

[6] U. Frisch, B. Hasslacher, and Y. Pomeau, Lattice-gas automata for the Navier-Stokes equations, Phys. Rev. Lett. 56 (14), 1505-1508 (1986).

[7] L. P. Kadanoff, G. R. McNamara, and G. Zanetti, A Poiseuille Viscometer for Lattice Gas Automata, Complex Systems 1, 791-803 (1987).

[8] S. N. Khotimah, I. Arif, The H. Liong, LatticeGas Cellular Automata Approach for Fluid Flow in Porous Media, Proceedings of Sixth International Conference on Knowledge-Based Intelligent Information \& Engineering Systems (KES'2002), IOS Press and Ohmsha, Damiani E. et.al.(Editor), Amsterdam, the Netherlands, 2002, pp.1512-1517.

[9] J. Šperl and J. Trčková, Permeability and Porosity of Rocks and Their Relationship Based on Laboratory Testing, Acta Geodyn. Geomater. 5(1), 41-47 (2008).

[10] L. I. Reyes, R. Parades V, and G. Gutierrez, Power law for the permeability in a twodimensional disordered porous medium, Physica A, 274, 391-399 (1999). 